\title{
A Study on Fatigue Crack Propagation of Rail Steel under Constant and Mixed Mode Variable Amplitude Loadings
}

\author{
Chul-Su Kim* and Kwang-Woo Chung ${ }^{\dagger}$
}

\begin{abstract}
Recently, axle load, operating speed and traffic density on railroads have had a tendency to increase and thereby cause additional pressure applied on used track. These operating conditions frequently result in service failure due to wear caused by wheel-rail contact and fatigue damage under cyclic loading. Among rail defects, the transverse crack, which has been the most dangerous type of fatigue damages, is developed from the subsurface crack near the rail running face and grows perpendicular to the rail surface. Therefore, it is necessary to investigate systematically the growth behavior of transverse crack for rail steel under mixed mode. In this study, the fatigue crack growth behavior of the transverse crack in rail steel was experimentally investigated under mixed-mode variable amplitude loadings.
\end{abstract}

Keywords : Comparative stress intensity factor range, Variable amplitude loading, Fatigue crack growth rate, Transverse crack

\section{Introduction}

Railway has advantages of high energy efficiency and environment friendly systems which represent the characteristics of punctuality, safety and mass transportation. However, the fatigue damages of rail steel are more frequently found in modern railways due to the accumulated impact on the rail which supports the whole axle load of the trains under severe environments from high traffic density as well as the recent speed upgrade of the lines. Among the various types of rail defects under cyclic loading from rail operation, the transverse crack is the most dangerous fatigue damage [1,2]. This starts normally from shelling, which is a subsurface crack near the rail running face, and it grows perpendicular to the rail surface. The fatigue crack normally develops a certain length horizontally and then grow vertically. Therefore the transverse crack was reported the most dangerous type of the fatigue cracks on rail because of the possibility of train derailment due to the broken rail [2]. To

\footnotetext{
Corresponding author: Department of Railway Operation System Engineering, Korea National University of Transportation

E-mail : ckw1201@ut.ac.kr

Department of Railway Vehicle System Engineering, Korea National University of Transportation
}

assure the safety of railway vehicles, it is necessary to investigate systematically fatigue crack growth behavior of rail steel under mixed mode loading.

The studies of the transverse crack on rail steel could be summarized as follows [1-4]. Kim et al. [2,3] investigated the characteristics of fatigue crack growth of rail steel and the gas pressure welded part of that under mixed mode constant amplitude loading. Desimone et al. [4] analyzed the fatigue crack propagation behavior of longitudinal flaws starting in the butt-welded joints of rails. Although these previous researches have been studied to understand fatigue crack growth (FCG) behavior under mode I constant amplitude loading, they could not evaluate the behavior of transverse crack propagation that undergoes mixedmode variable amplitude loading (VAL). Moreover, efforts to evaluate the fatigue crack growth behavior of rail steel under actual load in service have not been sufficiently carried out. In this study, the fatigue crack growth behavior of the transverse crack in rail steel was experimentally investigated under mixed-mode VAL.

\section{Experimental Procedure}

\subsection{Material and apparatus}

The material used in this study was $60 \mathrm{~kg}$ rail steel com- 


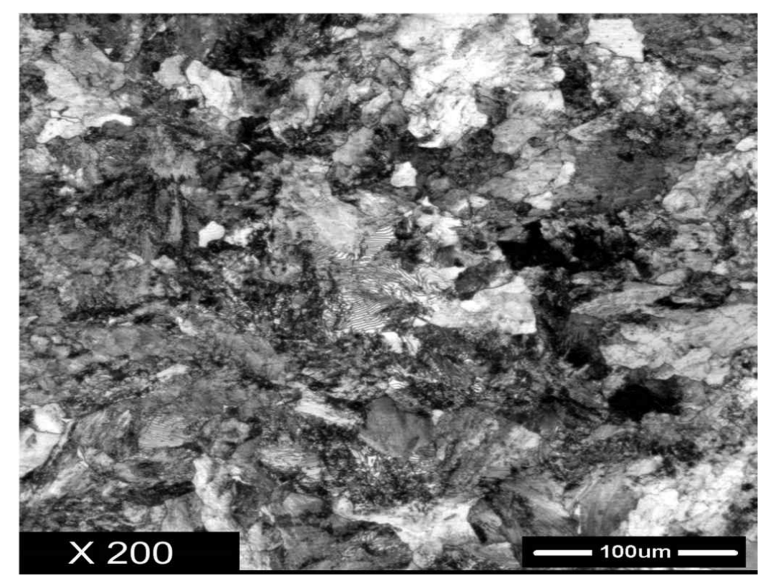

Fig. 1. Micrograph of $60 \mathrm{~kg}$ rail steel (200)

Table 1. Chemical composition of material (wt-\%)

\begin{tabular}{cccccccc}
\hline $\mathrm{C}$ & $\mathrm{Mn}$ & $\mathrm{P}$ & $\mathrm{S}$ & $\mathrm{Si}$ & $\mathrm{Ni}$ & $\mathrm{Cr}$ & $\mathrm{Mo}$ \\
\hline 0.721 & 0.873 & 0.012 & 0.005 & 0.256 & 0.032 & 0.053 & 0.011 \\
\hline
\end{tabular}

Table 2. Mechanical properties of material

\begin{tabular}{cccc}
\hline Yield strength & Tensile strength & Elongation & Hardness \\
\hline $481.2(\mathrm{MPa})$ & $887.1(\mathrm{MPa})$ & $14.5(\%)$ & $257.5(\mathrm{HV})$ \\
\hline
\end{tabular}

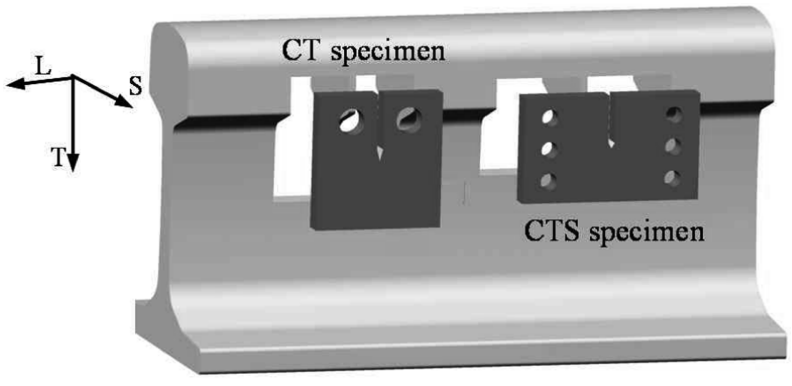

Fig. 2 CT and CTS specimens extracted from rail

monly used in Korean railroad track (INCHON IRON \& STEEL Co.). The microstructure of the rail consisted of a fully pearlitic structure as shown in Fig. 1.

The chemical composition and mechanical properties were summarized in Table 1 and 2, respectively. The types of fatigue specimen were CT and CTS (compact tension and shear) [2].

These specimens were cut from the rails in L-T orientation to model the behavior of transverse crack growth as shown in Fig. 2.

2.2 Fatigue crack growth test and comparative stress intensity factor

FCG (fatigue crack growth) test was carried out at room

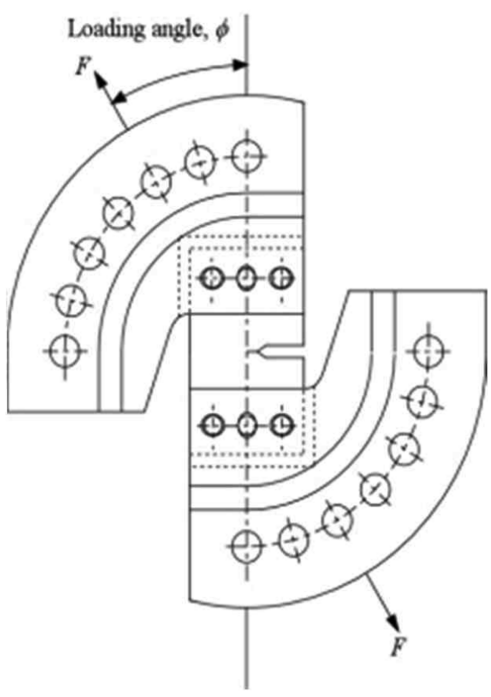

Fig. 3 Schematic representation of mixed-mode apparatus

temperature on a servo-hydraulic test machine (INSTRON, model 1332) having a load capacity of 25-tons with a frequency of $5 \mathrm{~Hz}$ under constant amplitude loading. These tests were conducted at stress ratio $R=0.05$

FCG test using CT specimen under mode I was performed according to ASTM E 647-95a. Until now, no unified fracture and fatigue test specifications or standards under mixed mode loadings have been established. Therefore, many different specimen geometries have been proposed in mixed mode crack growth studies [5] Experiments under mixed mode loadings in this study were conducted on a CTS specimen with a loading device as shown in Fig. 3.

This apparatus was based on the device proposed by Richard [6]. Precracking for CTS specimen was introduced under mode I loading with a sinusoidal waveform to an $a / W$ ratio of 0.55 , where $a$ and $W$ are the crack length and the width of the specimen respectively, using a stress ratio $R$ of 0 and a frequency of $8 \mathrm{~Hz}$. The various values of $K_{I} / K_{I I}$ could be achieved by changing the loading angle $\phi$ after introducing the fatigue precrack. The line of application of force $F$ extends at an angle $\phi$ to the longitudinal axis of the specimen. Depending on the size of angle $\phi$, mixed mode loadings occur at $0^{\circ}<\phi<90^{\circ}$. Fig. 4 shows the variation of stress intensity factors for the CTS specimen with the angle of applied load at aspect ratio $a / W=0.55$.

The direction of the fatigue crack under mixed mode loadings is not consistent with its initial direction. Therefore, under mixed mode loadings, it is important to evaluate not only the FCG rate but also the crack growth direction. To evaluate the characteristics of mixed mode fatigue crack, it is necessary to introduce the comparative stress intensity factor $K_{V}$ considering mode I and mode II 


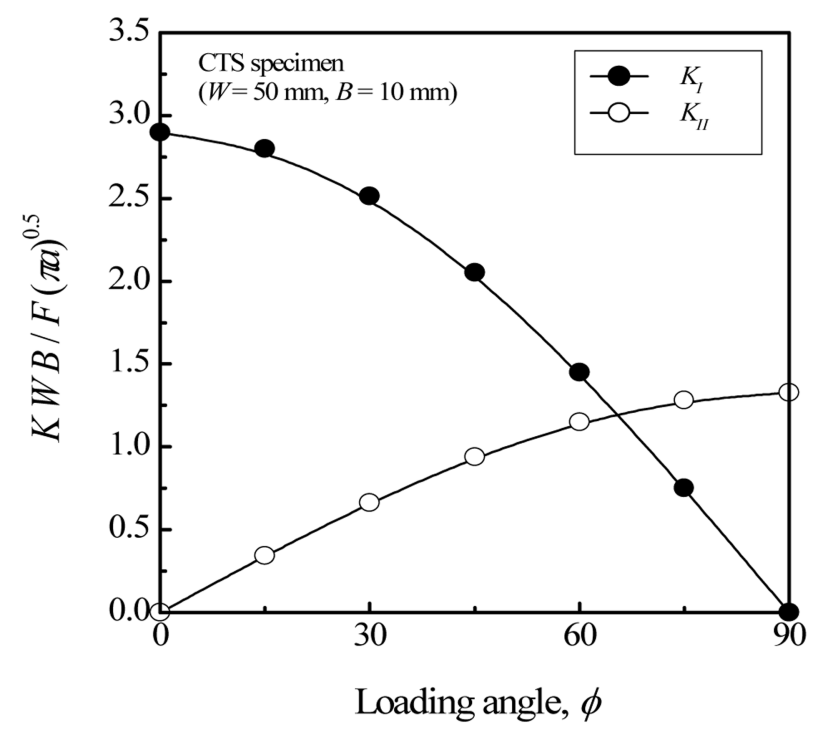

Fig. 4 Variation of stress intensity factors for the CTS specimen with angle of applied load

simultaneously.

Richard [5,6] proposed another comparative stress intensity factor $K_{V}$, with which the characteristic of material such as fracture toughness could be considered. The fatigue fractures under mixed mode occur when the following $K_{V}$ is equal to the fracture toughness $K_{I c}$.

$$
K_{V}\left(K_{I}, K_{I I}\right)=K_{I c}
$$

Based on the relationship between equation (1) and the mixed mode fracture boundary curve, the modified fracture boundary curve is proposed by Richard as shown in equation (2) [6]. $K_{V}$ in equation (1) could be expressed by equation (3).

$$
\begin{aligned}
& \left(\frac{K_{I}}{K_{I c}}\right)+\left(\zeta \frac{K_{I I}}{K_{I c}}\right)^{2}=1 \\
& K_{V}=\frac{1}{2} K_{I}+\frac{1}{2} \sqrt{K_{I}^{2}+4\left(\zeta K_{I I}\right)^{2}}
\end{aligned}
$$

where $\zeta$ denotes the fracture toughness ratio $\left(K_{I C} / K_{I I C}\right)$, which can be determined by the fracture boundary curve (ie. $K_{I I} / K_{I c}$ vs $K_{I} / K_{I c}$ ) with mixed mode experiments [6].

The $K_{I}$ and $K_{I I}$ for CTS specimen in the range from $0.5 \leq a / W \leq 0.7$ were computed using the finite element method as it shown in equation (4) $[5,6]$.

$$
K_{I}=\frac{F \sqrt{\pi a}}{W B} \frac{\cos \varphi}{1-\frac{a}{W}} \sqrt{\frac{0.26+2.65 \frac{a}{W-a}}{1+0.55 \frac{a}{W-a}-0.08\left(\frac{a}{W-a}\right)^{2}}}
$$

$$
K_{I I}=\frac{F \sqrt{\pi a}}{W B} \frac{\sin \varphi}{1-\frac{a}{W}} \sqrt{\frac{-0.23+1.40 \frac{a}{W-a}}{1-0.67 \frac{a}{W-a}+2.08\left(\frac{a}{W-a}\right)^{2}}}
$$

Where $F$ is the applied load, $B$ is the thickness of specimen, and $\phi$ is the applied loading angle.

The fatigue crack length and the direction of the crack growth were measured by a computerized image processing system under stroboscopic light illumination. A wave of variable amplitude loading was set one block, then the crack length and the crack path were measured at the end of every block. A 7-point incremental polynomial method was applied to determine the fatigue crack growth rate. Tests under mixed mode for the CTS specimen were conducted under constant amplitude and actual load at loading angle $\phi=30^{\circ}$ $\left(K_{I} / K_{V}=0.95\right)$ and $\phi=60^{\circ}\left(K_{I} / K_{V}=73\right)$, respectively.

\subsection{The evaluation of characteristic com- parative stress intensity factor (CCSIF).}

Barsom [8] proposed the root-mean-square(RMS) method to evaluate the stress intensity factor under mode I variable amplitude loading. RMS method could use root mean squares of stress history for equivalent constant amplitude loading in place of VAL to evaluate the stress intensity factor under VAL. The characteristic stress intensity factor $K_{r m s}$ can be expressed as;

$$
K_{r m s}=f(g) \sigma_{r m s} \sqrt{\pi a}, \sigma_{r m s}=\left[\frac{1}{n} \sum_{i=1}^{n}\left(\sigma_{i}\right)^{2}\right]^{1 / 2}
$$

where $\sigma_{i}$ is stress under VAL, $n$ is total number of stress cycles, $\sigma_{r m s}$ is equivalent characteristic stress, $f(g)$ is shape function, $K_{r m s}$ is RMS value of stress intensity factor under VAL and $a$ is crack length.

It is not available to define a certain stress intensity factor for under mixed mode VAL due to the load characteristics such as mode type and the variable amplitude. Therefore, to evaluate fatigue crack growth behavior under mixed-mode VAL, it is necessary to determine characteristic comparative stress intensity factor $K_{V, r m s}$, as given by equation (6), based on both $K_{V}$ proposed by Richard and the $K_{r m s}$ proposed by Barsom.

$$
K_{V, r m s}=\frac{1}{2} K_{I, r m s}+\frac{1}{2} \sqrt{K_{I, r m s}^{2}+4\left(\zeta K_{I I, r m s}\right)^{2}}
$$

\section{Results and Discussion}

\subsection{Fatigue crack growth behavior under constant amplitude loading}

The $K_{V}$ proposed by Richard under the mixed mode 


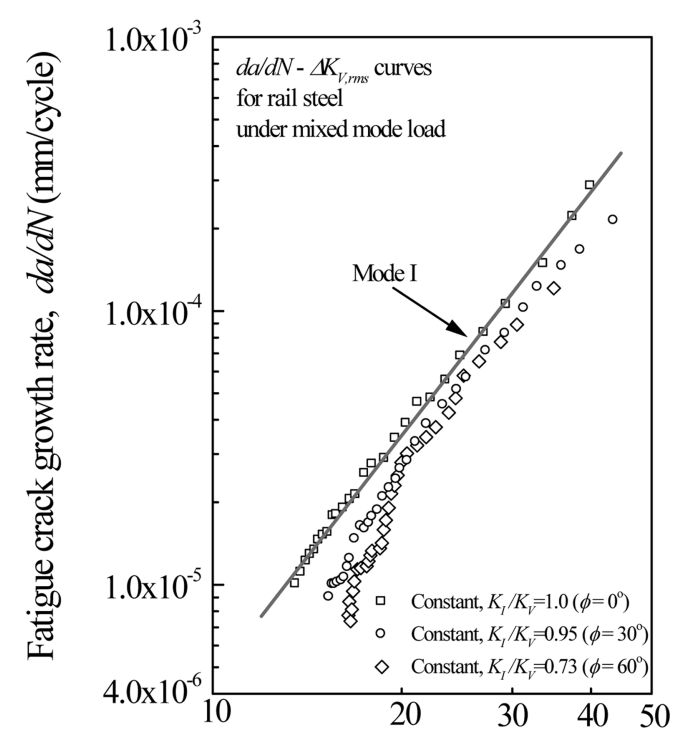

Stress intensity factor range, $K_{V}\left(\mathrm{MPa} \mathrm{m}^{1 / 2}\right)$

Fig. $5 d a / d N-\Delta K_{V}$ curves of rail steel under constant amplitude load with loading angle at $\mathrm{R}=0$

could be more appropriate for evaluating the FCG rate of rail steel [10], since the Richard model also considers the material characteristics such as fracture toughness. Fig. 5 shows the effect of mixed mode for fatigue crack growth behavior with the loading angle under constant amplitude loading at stress ratio $R=0$.

In the figure, rectangular mark $(\square)$ represents loading condition of mode I $\left(K_{I} / K_{V}=1\right)$, circle mark $(\bigcirc)$ represents $\phi=30^{\circ} \quad\left(K_{I} / K_{V}=0.95\right)$ and diamond mark $(\diamond)$ represents $\phi=60^{\circ}\left(K_{I} / K_{V}=73\right)$, respectively. From these figures, it is found that in the case of the low $\Delta K_{V}$ region, $d a / d N$ under the condition of mixed mode is slower than that of mode I. This difference is due to the effect of roughness-induced closure primarily from the $K_{I I}$ under mixed mode loadings [10].

\subsection{Fatigue crack growth behavior under actual load}

To assess the fatigue life of rail steel under the actual load in service, it is necessary to measure the actual load history of the rail. In order to obtain the real load history, a strain gauge was installed at the top of web part in the middle of tie in the longitudinal direction.

Fig. 6 shows the stress history $(\sigma)$ normalized by the yield strength $\left(\sigma_{y p}\right)$ of rail steel versus the number of reversals [9]. The total number of reversals in the stress history is 7282 (i.e. 1 block $=3641$ cycles) through the cycle counting method.

Fig. 7 shows the comparison between fatigue crack

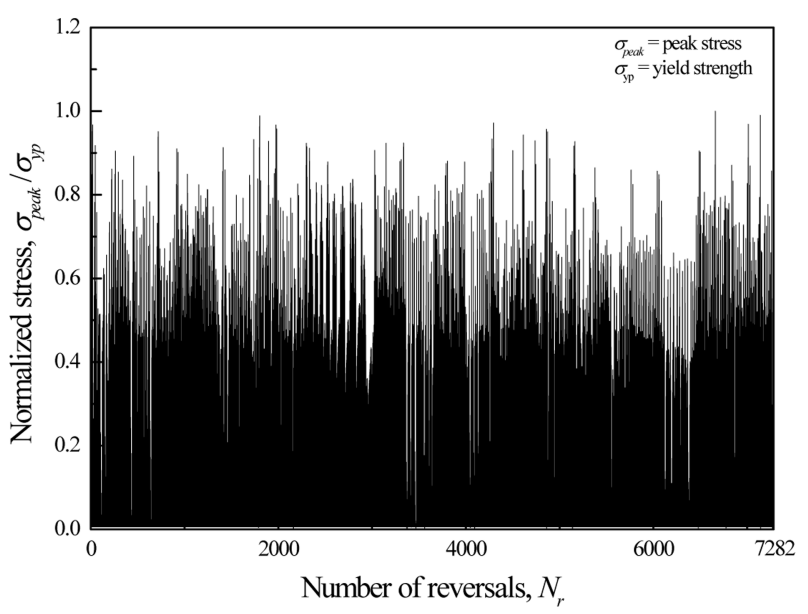

Fig. 6 Modified stress history in rail based on the actual load in service
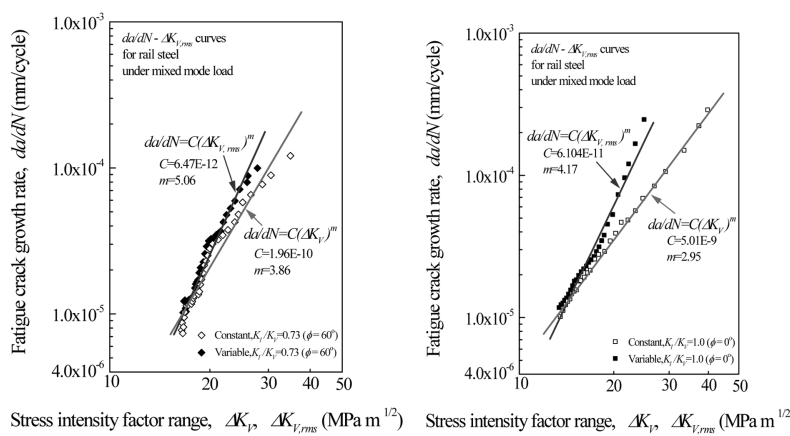

Fig. $7 d a / d N$ vs. $\Delta K_{V} \& \Delta K_{V, r m s}$ curves with loading angle

growth behaviors under constant amplitude and that of VAL. Solid marks $(\boldsymbol{\square}, \boldsymbol{\nabla})$ denote the case of constant amplitude, and open marks $(\square, \diamond)$ denote that of under VAL, respectively.

The fatigue crack growth rate $d a / d N$ under VAL shows a similar tendency with that of constant amplitude loading in low $\Delta K$ zone. However, the $d a / d N$ under VAL at loading angle $\phi=0^{\circ}$ shows the faster tendency than that of a constant amplitude when they move to high $\Delta K$ zone.

This result could be explained by two causes found in previous researches $[2,10]$. One is the crack closure effect by the roughness of crack surface and the other one is the size of plastic region which exists in the crack tip [2,10].

The first reason is the crack closure effect which is caused by mode II in mixed mode. It was reported that the crack closure phenomenon under mixed mode loading is related with the tortuous degree of the crack growth path and roughness of crack $[2,10]$. Linear-roughness index $[2,10]$, as shown in equation (7), is the result of the division of actual crack length $\left(L_{a}\right)$ by projected crack $\left(L_{p}\right)$, and ' $\lambda=1$ ' means the crack grows perpendicular to the loading direction. 


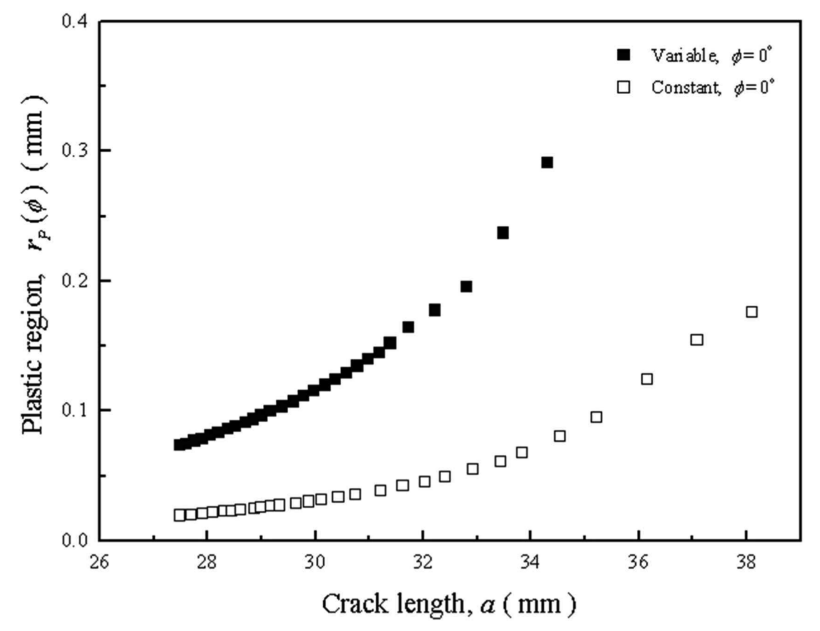

Fig. 8 Plastic zone size - crack length curves

$$
\lambda=L_{d} / L_{p}
$$

Where $L_{a}$ is actual crack length and $L_{p}$ is projected crack length.

Linear roughness index $\lambda$ under constant amplitude was $\lambda=1.06$ at $\phi=0$ and $\Delta K=20 \mathrm{MPa} \sqrt{\mathrm{m}}$, and that of VAL was $\lambda=1.16$. The value of $\lambda$ under VAL shows higher roughness than that of constant amplitude loading. Therefore fatigue crack growth rate under VAL should be evaluated as having a slower crack growth. On the contrary, $d a$ / $d N$ under VAL has the faster growth than that of constant amplitude over $\Delta K=20 \mathrm{MPa} \sqrt{\mathrm{m}}$ region. Previous researches [10] reported that the higher roughness index means the increase of crack closure effect and then the crack growth rate decrease. However, the experiment in this study showed different results in this region. Therefore, it is difficult to explain the cause that $d a / d N$ under VAL is greater than that of constant amplitude.

The difference of the plastic region size is the second reason which could explain the faster crack growth rate of VAL than that of constant amplitude. This could be explained by the effect of crack retardation and crack acceleration. The size of the plastic region of the crack tip under mixed mode constant amplitude and VAL can be determined by following equation.

$$
\begin{aligned}
& r_{p}(\phi)=\frac{1}{2 \pi \sigma_{y s}^{2}}\left\{K_{I}^{2} \cos ^{2} \frac{\phi}{2}\left[(1-2 v)^{2}+3 \sin ^{2} \frac{\phi}{2}\right]+K_{I} K_{I I} \sin \phi\right. \\
& \left.\times\left[3 \cos \phi-(1-2 v)^{2}\right]+K_{I I}^{2}\left[3+\sin ^{2} \frac{\phi}{2}(1-2 v)^{2}-9 \cos ^{2} \frac{\phi}{2}\right]\right\}
\end{aligned}
$$

Fig. 8 shows the relationship between crack length under mixed mode VAL and that of constant amplitude loading

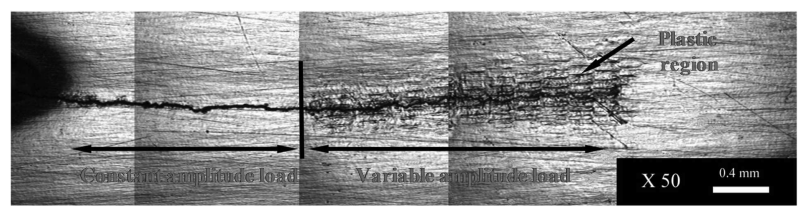

Fig. 9 Schematic illustration of plastic zone under constant amplitude and variable amplitude load

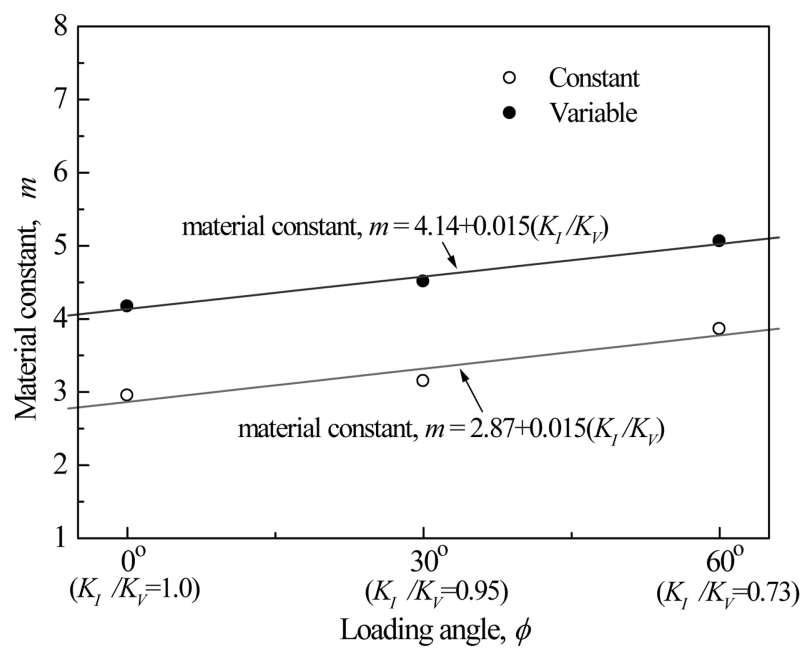

Fig. 10 Material constant - loading angle curves under constant amplitude load and variable amplitude load

at loading angle $\phi=0$ and plastic zone size.

In the figure, solid marks represent VAL, while open marks represent constant amplitude loading. Starting at an initial crack length of 27.5 for VAL and CAL(constant amplitude loading) the plastic region sizes were 0.07 and $0.02 \mathrm{~mm}$, respectively. After crack growth, it was found that CAL had a plastic region size of $0.2 \mathrm{~mm}$ at a crack length of $38 \mathrm{~mm}$, while VAL had a plastic region size of $0.3 \mathrm{~mm}$ at a crack length of $34 \mathrm{~mm}$. As crack length increases, so plastic size does for both CAL and VAL. However the increase was much greater for VAL than CAL. The plastic zone size measured shows the increasing tendency as the crack develops, and plastic zone size was longer in the case of VAL than of constant amplitude loading. Fig. 9 shows the schematic illustration of plastic zone under constant amplitude load and variable amplitude load.

Thus, it could be concluded that $d a / d N$ under VAL in the high $\Delta K_{V, r m s}$ region shows higher tendency than that of constant amplitude loading. This behavior was plotted using Paris' law, which represents material constants ( $c$ and $m$ ).

Fig. 10 shows the relationship between loading angle and material constant $m$, the sensitivity of $d a / d N$, under 
VAL and that of constant amplitude loading. Material constant $m$ under VAL is bigger than that of constant amplitude loading.

Moreover, $m$ under mixed mode is bigger than that of mode I.

\section{Conclusion}

In this study, the fatigue crack growth test of $60 \mathrm{~kg}$ rail steel used in Korea railway was experimentally investigated in order to evaluate the fatigue crack growth behavior under mixed- mode variable amplitude loadings based on the actual load history data. The following conclusions have been drawn.

1. To evaluate fatigue crack growth behavior under mixed-mode VAL, characteristic comparative stress intensity factor $K_{V, r m s}$ is proposed.

2. In the case of the low $\Delta K_{V}$ region, $d a / d N$ under the condition of mixed mode is slower than that of mode I. This difference is due to the effect of roughness-induced closure primarily from the $K_{I I}$ under mixed mode loadings.

3. The fatigue crack growth rate $d a / d N$ under VAL shows similar tendency with that under constant amplitude loading in low $\Delta K$ zone. However, the $d a / d N$ under VAL at loading angle $\phi=0$ shows the faster tendency than that of under constant amplitude when they move to high $\Delta K$ zone.

4. The plastic zone size measured shows the increasing tendency as the crack develops, and plastic zone size was longer in the case of VAL than that of constant amplitude loading. Therefore, $d a / d N$ under VAL in the high $\Delta K_{V, r m s}$ region shows higher tendency than that under constant amplitude loading.

5. Material constant $m$ (the sensitivity of $d a / d N$ ) under VAL is bigger than that of constant amplitude loading. Moreover, $m$ under mixed mode is bigger than that of mode I.

\section{References}

1. Orringer, O., Morris, J. M. and Jeong, D. Y. (1986). "Detail fracture growth in rails test results," Theoretical and Applied Fracture Mechanics, Vol. 5, pp. 63-95.

2. Kim, J. K. and Kim, C.S. (2002). "Fatigue crack growth behavior of rail steel under mode I and mixed mode loadings," Mat. Sci. \& Eng.(A)., Vol. 338, pp. 191-201.

3. Kim, C.S and Kim, J. K. (2007). "Fatigue crack growth behavior of a gas pressure welded part of rail steel under mixed mode loading," Key Eng. Mat., Vol. 345-346, pp.473476.

4. H. Desimone and S. Beretta (2006). "Mechanisms of mixed mode fatigue crack propagation at rail butt-welds," International Journal of Fatigue, Vol. 28, pp. 635-642.

5. Richard, H. A. (1987). "Crack problems under complex loading," Role of Fracture Mechanics in Modern Technology, pp. 577-588.

6. Richard, H. A. (1985). "Bruchvorhersage bei uberlagerter normal und schubbeansprunchung von rissen," VDI Forschungsheft 631. Disseldorf: VDI-Verlag, pp. 1-60.

7. Tong, J., Yates, J. R. and Brown, M. W. (1995). "A model for sliding mode crack closure, part II : Mixed mode I and II loading and application," Engineering Fracture Mechanics, Vol. 52, No. 4, pp. 613-623.

8. Barsom, E. M. (1973). "Fatigue crack growth under variable amplitude loading in ASTM A514 grade B steel," ASTM STP 536, American Society for Testing and Materials, Philadelphia.

9. Kim, C.S., Kim, J.K. and Kim, D.S. (2002). "Evaluation of fatigue strength and life in rail steel under variable amplitude load," JSME International Journal (A), Vol. 45, No. 4, pp. 495-503.

10. Tong, J., Yates, J. R. and Brown, M. W. (1995). "A model for sliding mode crack closure, part II : mixed mode I and II loading and application," Eng. Fract. Mech., Vol. 52, No. 4, pp. 613-623.

Received(April 14, 2012), Accepted(June 19, 2012) 